\title{
Correlation between serum lipid and prognosis of idiopathic sudden sensorineural hearing loss: a prospective cohort study
}

\author{
Minmin Shao, Guofeng Xiong, Guangzao Xiang, Shile Xu, Liqun Zhang \\ Department of Otorhinolaryngology, The Second Affiliated Hospital of Shanghai University (Wenzhou Central Hospital), Wenzhou, China \\ Contributions: (I) Conception and design: M Shao, L Zhang; (II) Administrative support: M Shao, L Zhang; (III) Provision of study materials or \\ patients: G Xiong, G Xiang; (IV) Collection and assembly of data: G Xiong, G Xiang, S Xu; (V) Data analysis and interpretation: M Shao, G Xiong; \\ (VI) Manuscript writing: All authors; (VII) Final approval of manuscript: All authors. \\ Correspondence to: Liqun Zhang. Department of Otorhinolaryngology, The Second Affiliated Hospital of Shanghai University, Wenzhou 325000, \\ China. Email: weyzlq@qq.com.
}

\begin{abstract}
Background: To investigate the correlation between blood lipids and the prognosis of idiopathic sudden sensorineural hearing loss (ISSNHL).

Methods: We included 232 patients with ISSNHL at the Second Affiliated Hospital of Shanghai University from June 2015 to March 2017 using a prospective cohort study design. We collected information including age, gender, hypertension, diabetes, mellitus, vertigo, as well as the levels of blood total cholesterol (TC), triglycerides (TG), and low-density lipoproteins (LDL-C). We also recorded the ratio between the levels of low-density lipoproteins and the levels of high-density lipoproteins (LDL-C/HDL-C ratio). Correlations between the prognosis of ISSNHL and TC, TG, LDL-C, and LDL-C/HDL-C ratio were analyzed by univariable and multivariable logistic regression analyses.

Results: The clinical effectiveness rate of patients with TC ranging from 5.2 to $6.2 \mathrm{mmol} / \mathrm{L}$ was significantly higher than that of patients with $\mathrm{TC}<5.2 \mathrm{mmol} / \mathrm{L}(\mathrm{P}<0.001)$. No notable difference was found between patients with TC $<5.2 \mathrm{mmol} / \mathrm{L}$ and patients with $\mathrm{TC} \geq 6.2 \mathrm{mmol} / \mathrm{L}$. The clinical effectiveness rate of patients in TG ranging from $1.7 \mathrm{mmol} / \mathrm{L}$ to $2.3 \mathrm{mmol} / \mathrm{L}$ was markedly higher than those in $\mathrm{TG}<1.7 \mathrm{mmol} / \mathrm{L}$ $(\mathrm{P}<0.001)$. No significant difference was found between patients with $\mathrm{TG}$ ranging from 2.3 to $5.6 \mathrm{mmol} / \mathrm{L}$, TG $\geq 5.6 \mathrm{mmol} / \mathrm{L}$, and TG $<1.7 \mathrm{mmol} / \mathrm{L}$. The clinical effectiveness rate of patients in LDL-C/HDL-C $<1.5$ was considerably higher than those in LDL-C/HDL-C ranging from 1.5 to $2.5,2.5$ to 3.5 , and $\geq 3.5$ $(\mathrm{P}<0.001)$.
\end{abstract}

Conclusions: Our findings indicated that TC, TG, and the LDL-C/HDL-C ratio are strongly associated with the prognosis of ISSNHL. These three indices could be recommended as independent markers to predict outcomes.

Keywords: Idiopathic sudden sensorineural hearing loss (ISSNHL); serum lipid; prognosis factor; prospective cohort study

Submitted Jan 21, 2021. Accepted for publication Apr 01, 2021.

doi: 10.21037/atm-21-907

View this article at: http://dx.doi.org/10.21037/atm-21-907

\section{Introduction}

Idiopathic sudden sensorineural hearing loss (ISSNHL) is an emergency in the department of otolaryngology. It has been defined as a loss of at least $30 \mathrm{~dB}$ over at least three frequencies in an audiogram within a 3-day period (1). The majority of cases are unilateral, while bilateral simultaneous or successive involvement accounts for $0.4-4.9 \%$ of all ISSHL cases (2). The presenting symptom is fullness or blocked ear, partly accompanied by tinnitus and dizziness $(3,4)$. The incidence in adults varies from 2-30 per 1,00,000. However, it has been reported that Germany has an 
Page 2 of 10

incidence of as high as 160 per 1,00,000 individuals per year $(5,6)$.

The prognostic factors of ISSNHL remain controversial. Some studies have proposed that the prognostic factors are varied, including the type of audiogram, the duration and severity of hearing loss, age, and accompanying symptoms (7). Meanwhile, other studies have found that hypertension, hypercholesterolemia, and hyperglycemia affect prognosis (8). Hyperlipemia is one of the risk factors of cardiovascular disease, which increases blood viscosity and alters vasomotion (9). Recent reports have identified hypercholesterolaemia as a significant risk factor for ISSNHL $(10,11)$. A systematic review and meta-analysis of the relationship between serum lipids and ISSNHL did not provide evidence for the association between them, nor did it definitively rule out such an association (12). Thus, additional studies are needed to ascertain the relationship, or lack thereof, between serum lipids and ISSNHL.

Given that the relationship between lipids and the prognosis of ISSNHL has not been confirmed, we aim to illuminate this relationship in this prospective cohort study.

We present the following article in accordance with the STROBE reporting checklist (available at http://dx.doi. org/10.21037/atm-21-907).

\section{Methods}

This is a prospective cohort study. The study was approved by the Ethics Committee of The Second Affiliated Hospital of Shanghai University. All procedures performed in this study involving human participants were in accordance with the Declaration of Helsinki (as revised in 2013). Informed consent was taken from all the patients.

\section{Setting}

This study was conducted in The Second Affiliated Hospital of Shanghai University.

\section{Participants}

We researched ISSNHL patients who were admitted to the Department of Otolaryngology at the Second Affiliated Hospital of Shanghai University from June 2015 to March 2017.

Patients were included based on the following criteria:

\section{Shao et al. Correlation between serum lipid and sudden deafness}

(I) meet the ISSNHL diagnostic criteria; (II) exclude other hearing loss disease including acoustic neuroma, cerebral stroke, otitis media, ossicular chain disruption, ceruminal impaction by auxiliary inspection (electro-otoscopy, pure tone checking, acoustic impedance and magnetic resonance imaging and auditory brainstem response).

Patients were excluded based on the following criteria: (I) onset time exceeding 7 days; (II) those with a history of surgery in the affected ear; and (III) those with accompanying middle ear disease in the affected ear.

\section{Data collection}

We collected the following patient data: gender, age, as well as accompanying vertigo, hypertension, and diabetes. Blood tests were performed in the early morning of the second day after hospitalization to test the following: total cholesterol (TC), triglycerides (TG), low-density lipoprotein cholesterol (LDL-C), and the low-density lipoprotein cholesterol/high-density lipoprotein cholesterol ratio (LDL-C/HDL-C ratio).

\section{Treatment}

Treatments consisted of high-dose steroids (intravenous administration of methylprednisolone sodium succinate $(60 \mathrm{mg})$ for 5 days and oral methylprednisolone tablets $(20 \mathrm{mg} /$ day) for another 3 days and vasoactive substances (Ginko Biloba Extract). After hospitalization, patients underwent a pure-tone audiogram (PTA) once every 5 days, and were followed up for 6 months after discharge. The patients were advised to have out-of-hospital rehabilitation including control cardiovascular risk factors, eating a light diet, avoiding fatigue and avoiding exposure to noisy environment. Patients who were followed up for less than one month were withdrawn from the study.

\section{Outcome assessment}

At the end of the follow-up period, the outcome assessment was based on the Clinical Practice Guideline: Sudden Hearing Loss from the American Academy of Otolaryngology-Head and Neck (1). The outcome was classified as either recovery or no recovery:

(I) Recovery (including partial and complete recovery): $>10-\mathrm{dB}$ improvement in PTA $(\mathrm{dB})$. 
(II) No recovery: $<10-\mathrm{dB}$ improvement in PTA (dB).

\section{Statistical analysis}

We used Stata 14.0(Stata Corp, America) for statistical analysis. Dichotomous variables were expressed as a percentage, and comparisons between the groups were determined by the chi-square test. Continuous variables were expressed as mean and standard deviation, and comparisons between the groups were determined by variance analysis. Logistic regressions were used to establish the correlation between TC, TG, LDL-C, and LDL-C/ HDL-C ratio and prognosis after adjustment for potential confounding factors (covariates included age, gender, hypertension, diabetes, vertigo, TC, TG, LDL-C, LDL-C/ HDL-C ratio). $\mathrm{P}$ values $<0.05$ were considered statistically significant.

\section{Results}

\section{Demographic characteristics of participants}

258 patients with ISSNHL were enrolled from June 2015 to March 2017. Follow-up was completed in 232 patients, and 26 patients were lost to follow-up and dropped out from the study. 1 patient withdrew due to a sudden outbreak of chickenpox, and the other 25 patients withdrew due to followed up for less than one month. Among 232 cases of ISSNHL, 113 cases (48.71\%) were in the right ear and 119 cases $(51.29 \%)$ were in the left ear. There were 105 males (45.3\%) and 127 females (54.7\%). Patients were classified into two groups (recovery and no recovery) according to their degree of hearing recovery. We analyzed the differences in demographic characteristics between participants in the two groups, including gender, age, diabetes hypertension, dizziness, and time from onset to treatment (Table 1). The differences in the time from onset, dizziness, and hypertension between the two groups were statistically significant $(\mathrm{P}<0.05)$.

\section{Serum lipid and prognosis}

To facilitate logistic analysis, serum TC, TG, LDL-C, and LDL-C/HDL-C were graded according to the Guidelines of the National Cholesterol Education Program (NCEP) Adult Treatment Panel III (ATP III) (Table 2) (13).

According to our analysis of the demographic characteristics, the time from onset, dizziness, and hypertension were found to affect prognosis. To eliminate the influence of these factors, univariate and multivariate analyses were performed. The adjusted covariates included age, sex, as well as the presence of hypertension, diabetes, and vertigo.

The recovery rate of patients in TC ranging from 5.2 to $6.2 \mathrm{mmol} / \mathrm{L}$ was significantly higher than that of patients in $\mathrm{TC}<5.2 \mathrm{mmol} / \mathrm{L}$ [univariable: odds ratio (OR) $=6.57,95 \%$ CI: 3.21-13.47, $\mathrm{P}<0.001$; multivariable-adjusted covariates: $\mathrm{OR}=8.49,95 \%$ CI: 3.16-22.80, $\mathrm{P}<0.001]$. No significant differences were observed between TC $\geq 6.2 \mathrm{mmol} / \mathrm{L}$ and TC $<5.2 \mathrm{mmol} / \mathrm{L}$ (univariable: $\mathrm{OR}=1.02,95 \% \mathrm{CI}: 0.49$ 1.93, $\mathrm{P}=0.946$; multivariable-adjusted covariates: $\mathrm{OR}=1.99$, 95\% CI: 0.59-6.79, P=0.270) (Table 3, Figure 1).

The recovery rate of patients in TG ranging from 1.7 to $2.3 \mathrm{mmol} / \mathrm{L}$ was notably higher than that of patients in TG $<1.7 \mathrm{mmol} / \mathrm{L}$ (univariable: $\mathrm{OR}=3.84,95 \%$ CI: $1.52-9.73$, $\mathrm{P}<0.05$; multivariable-adjusted covariates: $\mathrm{OR}=5.78,95 \%$ CI: $1.67-20.05, \mathrm{P}<0.05)$. There was no significant difference between patients in TG ranging from 2.3 to $5.6 \mathrm{mmol} / \mathrm{L}$ and $\mathrm{TG}<1.7 \mathrm{mmol} / \mathrm{L}$ (univariable: $\mathrm{OR}=0.59,95 \% \mathrm{CI}$ : 0.27-1.31, $\mathrm{P}=0.195$; multivariable-adjusted covariates: $\mathrm{OR}$ $=0.796,95 \%$ CI: $0.26-2.41, \mathrm{P}=0.687$ ), or between patients in $\mathrm{TG} \geq 5.6 \mathrm{mmol} / \mathrm{L}$ and $\mathrm{TG}<1.7 \mathrm{mmol} / \mathrm{L}$ (univariable: $\mathrm{OR}$ $=0.53$, 95\% CI: $0.19-1.50, \mathrm{P}=0.229$; multivariable-adjusted covariates: $\mathrm{OR}=0.67,95 \% \mathrm{CI}: 0.17-2.68, \mathrm{P}=0.573$ ) (Table 3, Figure 2).

The recovery rate patients in LDL-C/HDL-C $<1.5$ was significantly higher than patients in LDL-C/HDL-C ranging from 1.5 to 2.5 (univariable: $\mathrm{OR}=0.20,95 \% \mathrm{CI}$ : $0.07-0.60, \mathrm{P}<0.05$; multivariable-adjusted covariates: $\mathrm{OR}$ $=0.11,95 \%$ CI: $0.03-0.41, \mathrm{P}<0.05)$, LDL-C/HDL-C ranging from 2.5 to 3.5 (univariable: $\mathrm{OR}=0.25,95 \% \mathrm{CI}$ : $0.08-0.82, \mathrm{P}<0.05$; multivariable-adjusted covariates: OR $=0.16,95 \%$ CI: $0.04-0.68, \mathrm{P}<0.05)$, and LDL-C/HDL-C $\geq 3.5$ (univariable: $\mathrm{OR}=0.07,95 \%$ CI: $0.02-0.27, \mathrm{P}<0.001$; multivariable-adjusted covariates: $\mathrm{OR}=0.06,95 \% \mathrm{CI}: 0.01$ 0.39, $\mathrm{P}<0.05)$ (Table 3, Figure 3).

Our results showed that TC, TG, and LDL-C/HDL-C $(\mathrm{P}<0.05)$ affect prognosis in patients with ISSNHL. Curve fitting intuitively demonstrates the influence of blood lipids on prognosis. With the increase in TC levels, the effective rate of treatment showed a trend of initially increasing and then decreasing, with an overall inverted " $U$ " shape (Figure 4). Similarly, with the increase in TG levels, the effective rate of treatment showed a trend of initially increasing and then decreasing, with an overall inverted " $U$ " shape (Figure 5). Also, with the increase of LDL-C/HDL-C 
Table 1 Demographic characteristics of participants

\begin{tabular}{|c|c|c|c|c|}
\hline Variable & Numbers of recovery & Numbers of no recovery & OR value & $P$ value \\
\hline Male & 63 & 42 & 1 & \\
\hline Female & 79 & 48 & $0.911(0.536-2.356)$ & 0.732 \\
\hline \multicolumn{5}{|l|}{ Age (year) } \\
\hline $20-40$ & 29 & 15 & $0.467(0.088-2.483)$ & 0.372 \\
\hline $40-60$ & 74 & 42 & $0.440(0.089-2.171)$ & 0.314 \\
\hline$\geq 60$ & 32 & 31 & $0.258(0.051-1.312)$ & 0.103 \\
\hline \multicolumn{5}{|c|}{ Hypertension } \\
\hline \multicolumn{5}{|l|}{ Diabetes } \\
\hline Presence & 23 & 8 & 1 & \\
\hline Absence & 119 & 82 & $1.981(0.845-4.645)$ & 0.116 \\
\hline \multicolumn{5}{|l|}{ Dizziness } \\
\hline Presence & 14 & 27 & 1 & \\
\hline Absence & 128 & 63 & $0.255(0.125-0.520)$ & $<0.001^{*}$ \\
\hline \multicolumn{5}{|c|}{ Time from onset to treatment(days) } \\
\hline$<4$ & 58 & 22 & 1 & \\
\hline
\end{tabular}

${ }^{*}, \mathrm{P}<0.05$.

levels, the effective rate of treatment exhibited a trend of gradual decline (Figure 6).

\section{Discussion}

At present, the pathogenesis of ISSNHL is unclear. Viral infection, microcirculation disorder, autoimmune disease, cochlear membrane injury and stress are thought to be related to the onset of ISSNHL (14), and vascular endothelial dysfunction may also be involved (15). The structure and function of blood vessels, as well as the physicochemical properties of blood and hemorheology are the factors influencing normal blood flow. Abnormal blood or blood vessels can both lead to reduced blood flow, or even cause microcirculation disturbance (16). Abnormal blood lipid levels will result in changes in whole blood viscosity, which will lead to the disturbance of microcirculation and eventually to a lack of blood supply to target organs (17). If this occurs in the ear, it can lead to sudden sensorineural hearing loss.

Raised serum lipid levels increase the risk of cardiovascular disease and strokes (18). In recent years, the response-to-retention hypothesis of atherosclerosis has been gradually recognized. This hypothesis proposes that lipoprotein retention within the arterial wall is the key event in atherogenesis. The plasma concentration of LDL cholesterol must exceed $2 \mathrm{mmol} / \mathrm{L}$ for atherogenesis (19). In addition, coenzyme $\mathrm{Q}$ plays an important role in atherosclerosis. It can reverse many cellular changes of atherosclerosis, such as dysregulation of cholesterol biosynthesis, impaired autophagy flux, and mitochondrial dysfunction. Many studies have demonstrated that CoQ reduces macrophage accumulation, foam cell formation and lipid accumulation through reverse cholesterol 
Table 2 Classification of serum TC, TG, LDL-C, and LDL-C/ HDL-C ratio according to the NCEP ATP III

\begin{tabular}{|c|c|}
\hline Classification & Reference range \\
\hline \multicolumn{2}{|l|}{ Classification of TC (mmol/L) } \\
\hline Q1 (desirable) & $<5.2$ \\
\hline Q2 (borderline high) & $5.2-6.2$ \\
\hline Q3 (high) & $\geq 6.2$ \\
\hline \multicolumn{2}{|l|}{ Classification of TG (mmol/L) } \\
\hline Q1 (normal) & $<1.7$ \\
\hline Q2 (borderline high) & $1.7-2.3$ \\
\hline Q3 (high) & $2.3-5.6$ \\
\hline Q4 (very high) & $\geq 5.6$ \\
\hline \multicolumn{2}{|c|}{ Classification of LDL-C (mmol/L) } \\
\hline Q1 (optimal) & $<2.6$ \\
\hline Q2 (near or above optimal) & $2.6-3.4$ \\
\hline Q3 (borderline high) & $3.4-4.1$ \\
\hline Q4 (high) & $4.1-5.0$ \\
\hline Q5 (very high) & $\geq 5.0$ \\
\hline \multicolumn{2}{|l|}{ Classification of LDL-C/HDL-C } \\
\hline Q1 & $<1.5$ \\
\hline Q2 & $1.5-2.5$ \\
\hline Q3 & $2.5-3.5$ \\
\hline Q4 & $\geq 3.5$ \\
\hline
\end{tabular}

TC, total cholesterol; TG, triglycerides; LDL-C/HDL-C ratio, the ratio between the levels of low-density lipoproteins and the levels of high-density lipoproteins.

transport (RCT) and activate of AMP-activated protein kinase (AMPK). Cholesterol accumulation and high level of LDL-C leads to secondary CoQ deficiency and weaken the protective effect of coenzyme Q on atherosclerosis (20). Moreover, there are studies show that the patients were under a greater degree of stress before the onset of ISSHL, indicating that stress is associated with the onset of ISSHL. Stress stimulation can promote the adrenal cortex to secrete cortisol, resulting in an increase in the level of serum free fatty acids and the ability of the liver to produce TC. Therefore, TC also reflects the stress level of patients in 1 to 2 months (21).

In this study, TC, TG, LDL-C, and LDL-C/HDL-C were graded according to the NCEP-ATP III guidelines.
The results showed that ISSNHL patients with TC ranging from 5.2 to $6.2 \mathrm{mmol} / \mathrm{L}$ and TG ranging from 1.7 to $2.3 \mathrm{mmol} / \mathrm{L}$ have better prognosis. This suggests that the TC and TG levels are not positively or negatively correlated with the prognosis of ISSNHL. The prognosis was found to be optimal with TC levels ranging from 5.2 to $6.2 \mathrm{mmol} / \mathrm{L}$ and TG levels ranging from 1.7 to $2.3 \mathrm{mmol} / \mathrm{L}$.

We hypothesized that when TC $>6.2 \mathrm{mmol} / \mathrm{L}$ and TG $>2.3 \mathrm{mmol} / \mathrm{L}$, severe irreversible obstruction of the inner ear microvessels will lead to cochlear ischemia. If thrombolytic therapy misses the thrombolysis time window, the prognosis of patients is poor. When TC ranges from 5.2 to $6.2 \mathrm{mmol} / \mathrm{L}$ and $\mathrm{TG}$ ranges from 1.7 to $2.3 \mathrm{mmol} / \mathrm{L}$, hypercholesterolemia increases the whole blood viscosity of the inner ear microvessels, which leads to hemorheological alterations and incomplete ischemia. However, the damage is reversible and the prognosis is good. When TC $<5.2 \mathrm{mmol} / \mathrm{L}$ and TG $<1.7 \mathrm{mmol} / \mathrm{L}$, there may be other unspecified causes (such as an autoimmune reaction or viral infection) involved in the development of the disease. In these cases, the effectiveness of treatment is also poor.

Our study showed that the LDL-C/HDL-C ratio was an independent risk factor for ISSNHL after adjusting for confounding factors. There was a significant negative linear correlation between LDL-C/HDL-C ratio and recovery rate; the higher the ratio, the worse the prognosis. The potential mechanism of the LDL-C/HDL-C ratio influencing the prognosis of ISSNHL remains unclear; yet, we speculate that there are several possible mechanisms that explain this phenomenon. Cholesterol-rich LDL-C are directly implicated in the development of atherosclerotic cardiovascular disease (ASCVD), as evidenced by multiple studies (22). HDL-C is thought to be an atheroprotective cholesterol, which has several beneficial effects on the arterial vasculature (23). Thus, LDL-C/HDL-C ratio was a predictor for atherosclerosis. A high LDL-C/HDL-C ratio may indicate that atherosclerotic plaques are fragile and prone to rupture, ultimately leading to the appearance of thrombosis and resulting in vascular obstruction. In addition, LDL-C has been correlated with oxidative stress, and HDL-C has antioxidant effects $(24,25)$, and therefore, the LDL-C/HDL-C ratio is associated with inflammation. An increase in inflammatory components, or a decrease in anti-inflammatory and antioxidant components can lead to an increase in the LDL-C/HDL-C ratio. Inflammation and vascular embolization are considered to be possible mechanisms of ISSNHL, and the LDL-C/HDL-C ratio 
Table 3 Logistic regression analysis of related variables

\begin{tabular}{|c|c|c|c|c|}
\hline Variable & \multicolumn{2}{|c|}{ Univariate analysis } & \multicolumn{2}{|c|}{ Multivariate analysis } \\
\hline \multicolumn{5}{|c|}{$\mathrm{TC}(\mathrm{mmol} / \mathrm{L})$} \\
\hline$<5.2$ & 1 & & 1 & 0 \\
\hline $5.2-6.2$ & 6.574 (3.208-13.469) & 0.000 & 8.487 (3.160-22.797) & $<0.001$ \\
\hline \multicolumn{5}{|c|}{$\mathrm{TG}(\mathrm{mmol} / \mathrm{L})$} \\
\hline$<1.7$ & 1 & & 1 & \\
\hline $1.7-2.3$ & 3.842 (1.518-9.727) & 0.004 & $5.779(1.666-20.047)$ & 0.006 \\
\hline $2.3-5.6$ & $0.593(0.269-1.307)$ & 0.195 & $0.796(0.263-2.409)$ & 0.687 \\
\hline$<2.6$ & 1 & & 1 & \\
\hline $2.6-3.4$ & $1.470(0.760-2.845)$ & 0.253 & $0.566(0.220-1.456)$ & 0.238 \\
\hline $3.4-4.1$ & 3.429 (1.347-8.725) & 0.010 & $0.579(0.148-2.258)$ & 0.431 \\
\hline $4.1-5.0$ & $0.625(0.176-2.225)$ & 0.468 & $0.183(0.025-1.360)$ & 0.097 \\
\hline$\geq 5.0$ & $0.474(0.202-1.111)$ & 0.086 & $0.389(0.079-1.910)$ & 0.245 \\
\hline \multicolumn{5}{|c|}{ LDL-C/HDL-C } \\
\hline$<1.5$ & 1 & & 1 & \\
\hline $1.5-2.5$ & $0.197(0.065-0.595)$ & 0.004 & $0.110(0.030-0.412)$ & 0.001 \\
\hline
\end{tabular}

TC, total cholesterol; TG, triglycerides; LDL-C/HDL-C ratio, the ratio between the levels of low-density lipoproteins and the levels of highdensity lipoproteins.

\section{Study}

ID

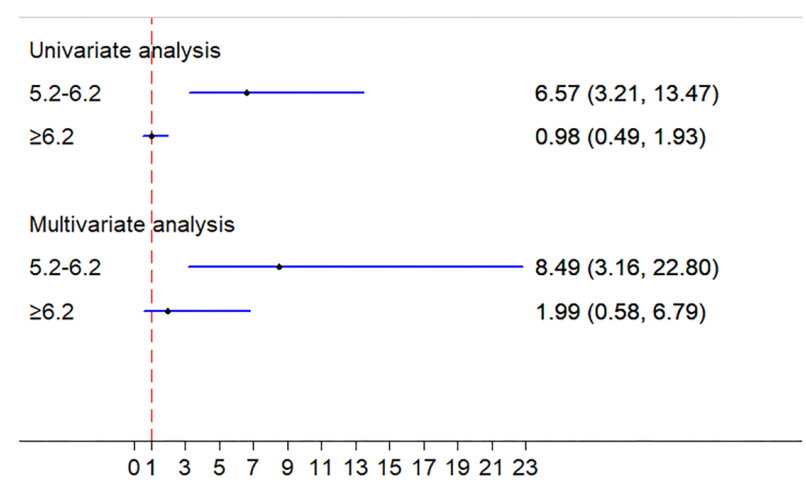

Figure 1 Univariate and multivariate analyses of prognosis between TC groups. TC, total cholesterol.

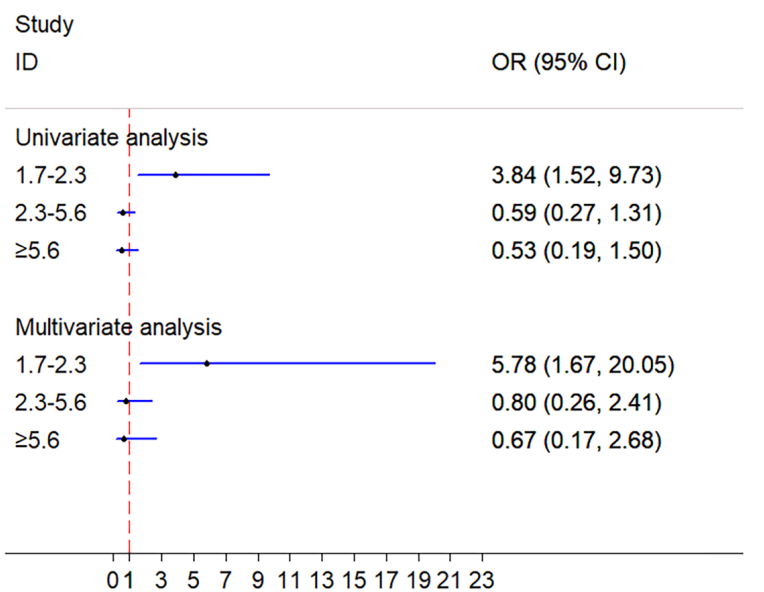

Figure 2 Univariate and multivariate analyses of prognosis between TG groups. TG, triglycerides. 
Study

ID

\section{Univariate analysis}

$1.5-2.5$

$2.5-3.5$

$\geq 3.5$.

Multivariate analysis

$1.5-2.5 \cdot$

$2.5-3.5$

$\geq 3.5$.

01305779011131517192123

Figure 3 Univariate and multivariate analyses of prognosis between LDL-C/HDL-C ratio groups. LDL-C/HDL-C ratio, the ratio between the levels of low-density lipoproteins and the levels of high-density lipoproteins.

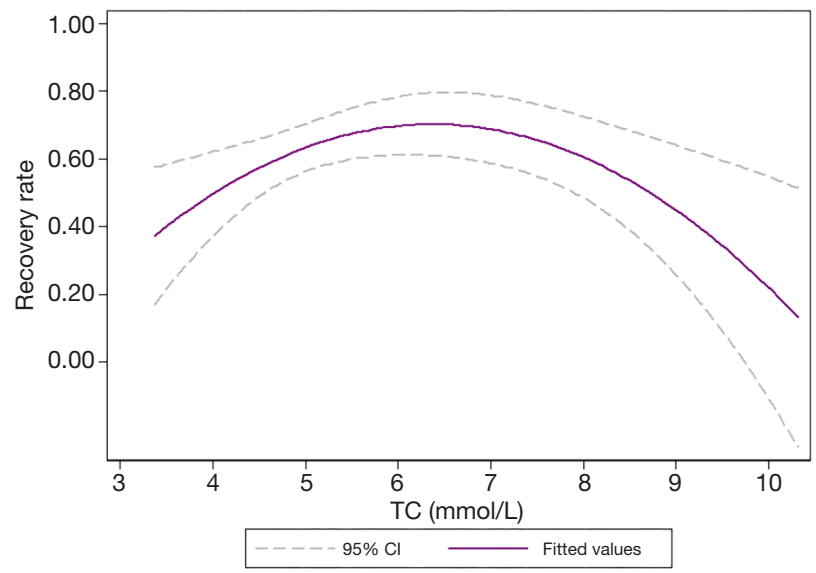

Figure 4 Curve fitting showed that with the increase in TC concentration, the recovery rate initially increased and then decreased, presenting an inverted " $U$ " shaped distribution. The middle purple line represented the fitted values, and the upper and lower dashed lines represented the $95 \%$ confidence intervals. TC, total cholesterol.

may reflect the severity of vascular embolization and inflammation, thus further impacting the prognosis.

Treatment of ISSNHL remains one of the most controversial issues in contemporary otolaryngology: although many countries have issued guidelines, the management of drug selection, dosage, time and route of administration is still not standardized. Systemic

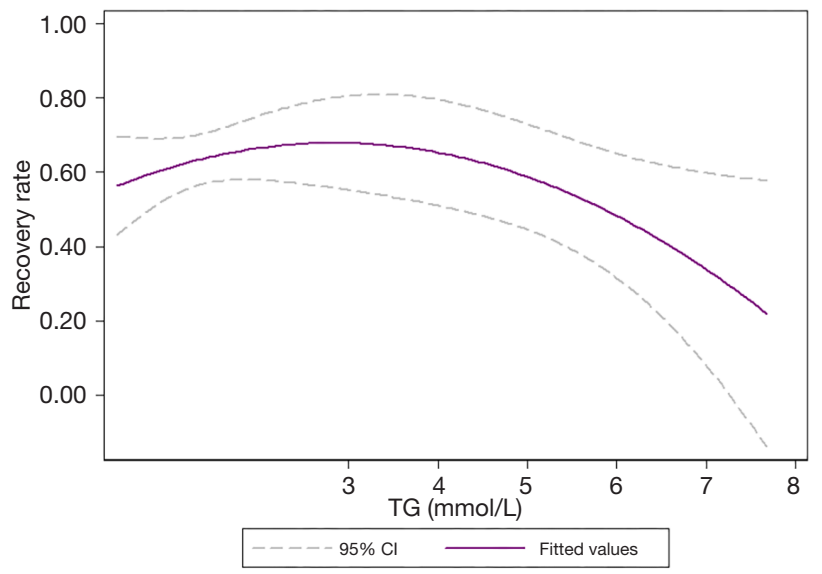

Figure 5 Curve fitting showed that with the increase in TG concentration, the recovery rate initially increased and then decreased, presenting an inverted "U" shaped distribution. The middle purple line represented the fitted values, and the upper and lower dashed lines represented the $95 \%$ confidence intervals. TG, triglycerides.

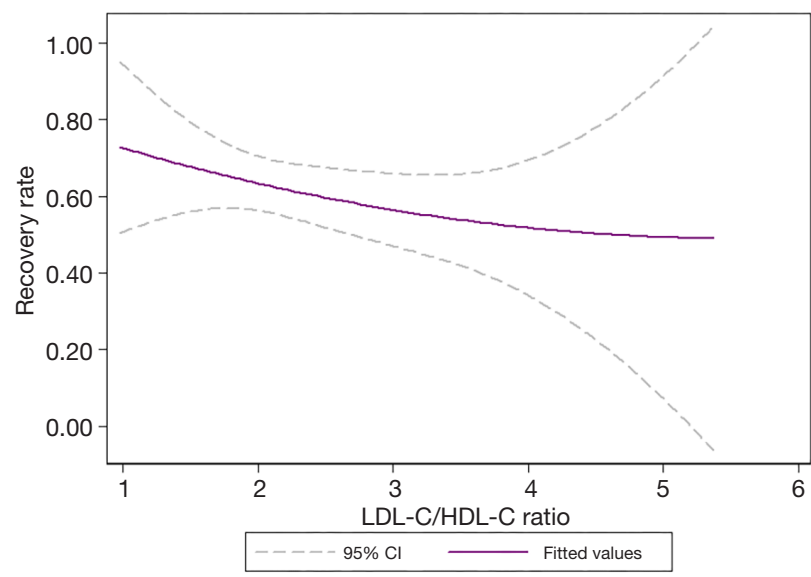

Figure 6 Curve fitting showed that with the increase in the LDL-C/HDL-C ratio, the recovery rate decreased. The middle purple line represented the fitted values, and the upper and lower dashed lines represented the $95 \%$ confidence intervals. LDL-C/HDL-C ratio, the ratio between the levels of low-density lipoproteins and the levels of high-density lipoproteins.

glucocorticoid is widely used standard primary treatment for ISSNHL which is recommended used whin 2 weeks of onset (1). To avoid the adverse drug reactive of systemic glucocorticoid in some patients and given the theoretical possibility of achieving higher blood concentrations in the 


\section{Page 8 of 10}

inner ear, intratympanic steroid therapy may be used as a primary therapy alone or as a salvage therapy after failure of systemic steroids (26). As a salvage therapy, intratympanic steroid therapy should be given in 2 to 6 weeks of onset. However, the therapeutic effect of steroids is not always satisfactory. To avoid ineffective treatment and associated risks, complications side effects, costs, and potential adverse interactions with effective therapies, antivirals, thrombolytics, vasodilators, or vasoactive substances is not routinely used. It should be used appropriately in individualized treatment according to the condition. Ginko Biloba Extract (GBE) is recommended by Chinese Guidelines for diagnosis and treatment of ISSNHL. It contains flavones and terpenes, prevents the development of free radicals in cases of ischemic injury and also prevents vascular contraction. Studies have shown that GBE can improve the mean auditory threshold and the symptoms of tinnitus and dizziness (27).

\section{Limitations}

Firstly, due to the lack of patients under 8 years old of age in this study, our research result is generalisable to the post-school population. Secondly, there were a certain percentage of spontaneous recovery rates of ISSNHL, which could not be excluded in this study. Thirdly, this study is an observational cohort study, as opposed to a clinical randomized controlled trial, and there were potential differences in patient baseline characteristics between the two groups, which will affect the statistical results.

\section{Conclusions}

Our findings indicated that hypertension, dizziness, time from onset to treatment, TC, TG, and LDL-C/HDL-C ratio are strongly associated with the prognosis of ISSNHL. When patients are accompanied by hypertension, dizziness, and a long time from onset to treatment, their prognosis will be worse. Age, diabetes and gender is not the prognosis factor of ISSNHL. TC, TG, LDL-C/HDL-C ratio could be recommended as independent markers to predict outcomes. When TC ranges from 5.2 to $6.2 \mathrm{mmol} / \mathrm{L}$, TG ranges from 1.7 to $2.3 \mathrm{mmol} / \mathrm{L}$, or the LDL-C/HDL-C ratio is low, this suggests that the prognosis of patients with SSNHL will be better; otherwise, the prognosis will be worse.

\section{Shao et al. Correlation between serum lipid and sudden deafness}

\section{Acknowledgments}

Funding: None.

\section{Footnote}

Reporting Checklist: The authors have completed the STROBE reporting checklist. Available at http://dx.doi. org/10.21037/atm-21-907

Data Sharing Statement: Available at http://dx.doi. org/10.21037/atm-21-907

Conflicts of Interest: All authors have completed the ICMJE uniform disclosure form (available at http://dx.doi. org/10.21037/atm-21-907). The authors have no conflicts of interest to declare.

Ethical Statement: The authors are accountable for all aspects of the work in ensuring that questions related to the accuracy or integrity of any part of the work are appropriately investigated and resolved. The study was approved by the Ethics Committee of The Second Affiliated Hospital of Shanghai University. All procedures performed in this study involving human participants were in accordance with the Declaration of Helsinki (as revised in 2013). Informed consent was taken from all the patients.

Open Access Statement: This is an Open Access article distributed in accordance with the Creative Commons Attribution-NonCommercial-NoDerivs 4.0 International License (CC BY-NC-ND 4.0), which permits the noncommercial replication and distribution of the article with the strict proviso that no changes or edits are made and the original work is properly cited (including links to both the formal publication through the relevant DOI and the license). See: https://creativecommons.org/licenses/by-nc$\mathrm{nd} / 4.0 \%$.

\section{References}

1. Chandrasekhar SS, Tsai Do BS, Schwartz SR, et al. Clinical Practice Guideline: Sudden Hearing Loss (Update). Otolaryngol Head Neck Surg 2019;161:S1-45.

2. Bing D, Wang DY, Lan L, et al. Comparison between Bilateral and Unilateral Sudden Sensorineural Hearing Loss. Chin Med J (Engl) 2018;131:307-15. 
3. Lee JY, Lee YW, Chang SO, et al. Vestibular function analysis of sudden sensorineural hearing loss with dizziness. J Vestib Res 2020;30:203-12.

4. Nogueira-Neto FB, Gallardo FP, Suzuki FA, et al. Prognostic and Evolutive Factors of Tinnitus Triggered by Sudden Sensorineural Hearing Loss. Otol Neurotol 2016;37:627-33.

5. Singh A, Kumar Irugu DV. Sudden sensorineural hearing loss - A contemporary review of management issues. J Otol 2020;15:67-73.

6. Kleyn AD. Sudden complete or partial loss of function of the octavus-system in apparently normal persons. Acta Otolaryngol 1944;32:407-29.

7. Kang WS, Yang CJ, Shim M, et al. Prognostic Factors for Recovery from Sudden Sensorineural Hearing Loss: A Retrospective Study. J Audiol Otol 2017;21:9-15.

8. Lin CF, Lee KJ, Yu SS, et al. Effect of comorbid diabetes and hypercholesterolemia on the prognosis of idiopathic sudden sensorineural hearing loss. Laryngoscope 2016;126:142-9.

9. Flora GD, Nayak MK. A Brief Review of Cardiovascular Diseases, Associated Risk Factors and Current Treatment Regimes. Curr Pharm Des 2019;25:4063-84.

10. Lin HC, Wang CH, Chou YC, et al. The correlation between lipoprotein ratios and hearing outcome in idiopathic sudden sensorineural hearing loss patients. Clin Otolaryngol 2015;40:355-62.

11. Sciancalepore PI, de Robertis V, Sardone R, et al. Sudden sensorineural hearing loss: What factors influence the response to therapy? Audiol Res 2020;10:234.

12. Chang IJ, Kang CJ, Yueh CY, et al. The relationship between serum lipids and sudden sensorineural hearing loss: a systematic review and meta-analysis. PLoS One 2015;10:e0121025.

13. Expert Panel on Detection, Evaluation, and Treatment of High Blood Cholesterol in Adults. Executive Summary of The Third Report of The National Cholesterol Education Program (NCEP) Expert Panel on Detection, Evaluation, And Treatment of High Blood Cholesterol In Adults (Adult Treatment Panel III). JAMA 2001;285:2486-97.

14. Yigider AP, Keskin M, Kufeciler L, et al. Topography of the lesion in idiopathic sudden sensorineural hearing loss. Acta Otolaryngol 2020;140:909-13.

15. Gündoğan F, Bayram A, Kalkan M, et al. Plasma levels of endothelial cell-specific molecule- 1 and pentraxin-3 in idiopathic sudden sensorineural hearing loss. J Laryngol Otol 2018;132:995-9.

16. Mitrophanov AY, Govindarajan V, Zhu S, et al. Microfluidic and computational study of structural properties and resistance to flow of blood clots under arterial shear. Biomech Model Mechanobiol 2019;18:1461-74.

17. Tripolino C, Irace C, Carallo C, et al. Body fat and blood rheology: Evaluation of the association between different adiposity indices and blood viscosity. Clin Hemorheol Microcirc 2017;65:241-8.

18. Wang HH, Garruti G, Liu M, et al. Cholesterol and Lipoprotein Metabolism and Atherosclerosis: Recent Advances in Reverse Cholesterol Transport. Ann Hepatol 2017;16 Suppl 1:S27-42.

19. Williams KJ, Tabas I. The response-to-retention hypothesis of early atherogenesis. Arterioscler Thromb Vasc Biol 1995;15:551-61.

20. Suárez-Rivero JM, Pastor-Maldonado CJ, de la Mata M, et al. Atherosclerosis and Coenzyme Q10. Int J Mol Sci 2019;20:5195.

21. Watanabe H, Sano H, Maki A, et al. Investigation of Stress Levels before the Onset of Idiopathic Sudden Sensorineural Hearing Loss. J Int Adv Otol 2019;15:51-5.

22. Ference BA, Ginsberg HN, Graham I, et al. Low-density lipoproteins cause atherosclerotic cardiovascular disease 1. Evidence from genetic, epidemiologic, and clinical studies. A consensus statement from the European Atherosclerosis Society Consensus Panel. Eur Heart J 2017;38:2459-72.

23. Smit RA, Jukema JW, Trompet S. Increasing HDL-C levels with medication: current perspectives. Curr Opin Lipidol 2017;28:361-6.

24. Oliveira JVB, Lima RPA, Pordeus Luna RC, et al. The direct correlation between oxidative stress and LDL-C levels in adults is maintained by the Friedewald and Martin equations, but the methylation levels in the MTHFR and ADRB3 genes differ. PLoS One 2020;15:e0239989.

25. Hofmanis J, Hofmane D, Svirskis S, et al. HDL-C Role in Acquired Aortic Valve Stenosis Patients and Its Relationship with Oxidative Stress. Medicina (Kaunas) 2019;55:416.

26. Marx M, Younes E, Chandrasekhar SS, et al. International consensus (ICON) on treatment of sudden sensorineural hearing loss. Eur Ann Otorhinolaryngol Head Neck Dis 2018;135:S23-8. 
Page 10 of 10

27. Koo JW, Chang MY, Yun SC, et al. The efficacy and safety of systemic injection of Ginkgo biloba extract, EGb761, in idiopathic sudden sensorineural hearing loss: a randomized placebo-controlled clinical trial. Eur Arch

Cite this article as: Shao M, Xiong G, Xiang G, Xu S, Zhang L. Correlation between serum lipid and prognosis of idiopathic sudden sensorineural hearing loss: a prospective cohort study. Ann Transl Med 2021;9(8):676. doi: 10.21037/atm-21-907

\section{Shao et al. Correlation between serum lipid and sudden deafness}

Otorhinolaryngol 2016;273:2433-41.

(English Language Editor: A. Kassem) 